\title{
Microbial production of value-added nutraceuticals
}

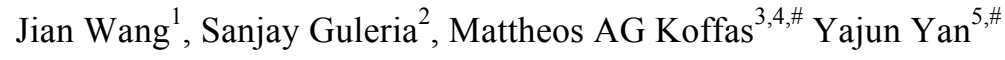

\section{Addresses}

${ }^{1}$ College of Engineering, University of Georgia, Athens, Georgia 30602, United States

${ }^{2}$ Division of Biochemistry, Sher-e-Kashmir University of Agricultural Sciences and Technology, Main Campus Chatha-180009, Jammu, India

3 Department of Chemical and Biological Engineering, Center for Biotechnology and Interdisciplinary Studies (CBIS), Rensselaer Polytechnic Institute, $1108^{\text {th }}$ Street, Troy, NY 12180, United States

${ }^{4}$ Department of Biology, Center for Biotechnology and Interdisciplinary Studies (CBIS), Rensselaer Polytechnic Institute, $1108^{\text {th }}$ Street, Troy, NY 12180, United States

${ }^{5}$ BioChemical Engineering Program, College of Engineering, University of Georgia, Athens, Georgia 30602, United States

${ }^{\#}$ Co-corresponding authors Koffas, Mattheos AG (koffam@rpi.edu)

Yan, Yajun (yajunyan@uga.edu)

\begin{abstract}
Nutraceuticals are important natural bioactive compounds that confer health-promoting and medical benefits to humans. Globally growing demands for value-added nutraceuticals for prevention and treatment of human diseases have rendered nutraceuticals a multi-billion dollar market. However, supply limitations and extraction difficulties from natural sources such as plants, animals or fungi, restrict the large-scale use of nutraceuticals. Metabolic engineering via microbial production platforms has been advanced as an eco-friendly alternative approach for production of value-added nutraceuticals from simple carbon sources. Microbial platforms like the most widely used Escherichia coli and Saccharomyces cerevisiae have been engineered as versatile cell factories for production of diverse and complex value-added chemicals such as phytochemicals, prebiotics, polysaccaharides and poly amino acids. This review highlights the recent progresses in biological production of value-added nutraceuticals via metabolic engineering approaches.
\end{abstract}

\section{Introduction}

Nutraceutical, a hybrid term from "nutrition" and "pharmaceutical" coined by Stephen L. DeFelice in 1989, was defined as "any substance that is a food or a part of food and provides medical or health benefits, including the prevention and treatment of disease" [1]. Later on, the concept of nutraceutical was modified as "a product isolated or purified from foods that is generally sold in medicinal forms not usually associated with food" [2]. Nutraceuticals are now more specific to structurally and functionally diverse bioactive compounds that exert long-tern medicinal or physiological benefits other than purely nutritional or direct pharmaceutical effects, which distinguishes them from functional foods and drugs. They can be derived from plants (e.g. 
phytochemicals, vitamins), from animals (e.g. polysaccharides), from microorganisms (e.g. poly amino acids) and from marine sources (e.g. glucosamine and chitosan). Nutraceuticals are widely used for their health-promoting or disease-preventing properties especially in preventing aging-associated diseases including oxidative stress, depression, inflammation, arthritis, osteoporosis, gastrointestinal diseases, cardiovascular diseases, diabetes and cancer [3]. The rising demands and interests in maintaining human wellness through diet have greatly promoted the growth of the nutraceutical market. According to Global Information Inc.' recent report, the global nutraceutical market was estimated to exceed $\$ 171.8$ billion in 2014 and reach $\$ 241.1$ billion by 2019, while the US market alone was about $\$ 75.9$ billion [3].

However, the expanding nutraceutical market can hardly be fulfilled by the productivity of conventional nutraceutical industries. Direct extraction strategies are limited by the availability and cost of raw materials, the quality control of supplies, and the low content and purity of nutraceuticals. Although chemical synthesis is an alternative approach, it is limited to producing simple biochemicals and is infeasible for complex biochemicals especially those that are chemically unfavorable [4]. To address these issues, microbial-based metabolic engineering is an appealing approach and has achieved great progress on production of value-added nutraceuticals in very recent years. The rapid elucidation of biosynthetic pathways for natural products and the genetic amenability of microorganisms have enabled the development of microbial hosts for production of various nutraceuticals. By further genetic manipulation of host cells and optimization of the culture conditions or fermentation processes, metabolic engineering makes it possible to scale up the production of nutraceuticals from simple carbon sources. As a matter of fact, certain commercial E. coli strains and food-grade S. cerevisiae have been industrially used as cell factories to produce natural products Generally Recognized As Safe (GRAS). This review covers recent advances in nutraceutical production via microbial-based metabolic engineering, including the well-studied phytochemicals, as well as prebiotics, polysaccaharides and poly amino acids.

\section{Phytochemicals}

Phytochemicals are a broad spectrum of plant-derived bioactive secondary metabolites that are commonly found in fruits, vegetables, beans and grains. They are involved in plant defenses against biotic or abiotic stresses, and also exhibit health-protecting or disease-preventing effects on humans [3]. Phytochemicals is a vast and very important repertoire for nutraceuticals, which include polyphenolic compounds (flavonoids, isoflavonoids, stilbenoids and curcuminoids), alkaloids, terpenoids (monoterpenes, diterpenes, tetraterpenes, polyterpenes, steroid saponins, lycopene and carotenoids) and their derived compounds.

\section{Polyphenolic compounds}

Amongst the phytochemicals, polyphenolic compounds have been used for decades as effective antioxidants and food ingredients in the nutraceutical industry. Polyphenolic scaffolds such as flavonoids and stilbenoids are derived from the phenylpropanoic pathway that extends from aromatic amino acids L-phenylalanine and L-tyrosine (Figure 1) [5]. L-phenylalanine and L-tyrosine are converted to pivotal intermediates cinnamic acid and $p$-coumaric acid via phenylalanine ammonia lyase (PAL) and tyrosine ammonia lyase (TAL), which are further converted to cinnamoyl-CoA and $p$-coumaroyl-CoA by 4-coumaroyl-CoA ligase (4CL), 
respectively. Then, three moles of malonyl-CoA condense with cinnamoyl-CoA or $p$-coumaroyl-CoA to construct the backbone flavanones, stibenes or curcuminoids by type III polyketide synthases, such as chalcone synthase (CHS), curcuminoid synthase (CUS) or stilbene synthase (STS) [6]. With the action of tailoring enzymes such as hydroxylases, $O$-methyltransferases, glucosyltransferase, lipases and prenyltransferase, diverse polyphenolic compounds are generated [7].

$E$. coli and $S$. cerevisiae have generally been developed as platform organisms for de novo or semi-de novo production of almost all kinds of polyphenolic compounds (Table 1). Some of the biggest advantages of $E$. coli include its fast growth and ease of genetic manipulation; for $S$. cerevisiae its Generally Regarded As Safe (GRAS) status and its ability to functionally express plant metabolic enzymes. In all cases, in order to achieve polyphenolic compound production in microbes, plant-originated enzymes are firstly overexpressed via either codon optimization or construction of enzyme chimeras that facilitate bacterial expression [8]. The first strategy is feeding precursors to the heterologous hosts to produce corresponding polyphenolic compounds. For example, supplementation with phenylalanine to E. coli expressing heterologous pathways produced chrysin $(9.4 \mathrm{mg} / \mathrm{L})$ or galangin $(1.1 \mathrm{mg} / \mathrm{L})$ while supplementation with tyrosine led to the production of apigenin $(13 \mathrm{mg} / \mathrm{L})$ or kaempferol $(15.1 \mathrm{mg} / \mathrm{L})$ (Table 1) [9]. This feeding approach is widely used when precursors are low or cannot be biosynthesized in microbes. Flavonoid glucosides like luteolin 4'-O- and 7'- $O$-glucosides were produced by feeding luteolin to E. coli expressing glycosyltransferases UGT71G1 [10]. By expressing glycosyltransferases with different substrate specificities, quercetin 3-O-glucoside, quercetin-3-O-glucuronide, quercetin 3-O-galactoside and quercetin-3-O-rhamnoside were respectively produced (Table 1) [10-12]. The second approach is de novo biosynthesis of phenolic compounds from simple carbon sources like glucose and glycerol, which is more appealing for industrial applications. E. coli hosts can be easily metabolically engineered to overproduce L-phenylalanine and L-tyrosine from simple carbon sources. The intracellular concentration of malonyl-CoA, the extender unit for polyphenolic backbones, can be greatly enhanced in E. coli by overexpressing acetyl-CoA carboxylase (ACC) or genome-scale metabolic optimization or by repressing malonyl-CoA consumption pathways using antisense RNA [13,14]. E. coli has been successfully utilized for de novo biosynthesis of certain polyphenolic compounds such as pinocembrin, pinosylvin, sakuranetin and ponciretin [15-17]. Furthermore, substrate promiscuity of authentic or engineered enzymes has provided with new possibilities to produce novel or non-natural polyphenolic compounds [18,19].

\section{Alkaloids}

Alkaloids are amino acid-derived nitrogenous compounds that have important therapeutic values, including anticancer and antimalarial effects [5]. For a long time, production of alkaloids was limited to plants because of their complex structure and long biosynthetic pathways. The three most well-known alkaloids are benzylisoquinoline alkaloids (BIAs) derived from tyrosine, monoterpene indole alkaloids (MIAs) derived from tryptophan and glucosinolates. In recent years, elucidation of many BIA biosynthetic pathways enabled the reconstruction of BIA pathways in both E. coli and S. cerevisiae [20-22]. (S)-reticuline, a branch-point intermediate for BIA, was biosynthesized from simple carbon sources with titer of $46.0 \mathrm{mg} / \mathrm{L}$ [20]. Bacteria consortia of $E$. coli producing $(S)$-reticuline and $S$. cerevisiae producing either monooxygenase CYP80G2 or the berberine bridge enzyme (BBE) yielded $7.2 \mathrm{mg} / \mathrm{L}$ magnoflorine and $8.3 \mathrm{mg} / \mathrm{L}$ scoulerine, 
respectively (Figure1) [23]. S. cerevisiae was also enabled to produce $(R, S)$-reticuline and was further engineered to generate scoulerine, tetrahydrocolumbamine and tetrahydroberberine from $(S)$-reticuline and salutaridine from $(R)$-reticuline [24]. However, metabolic engineering of MIA alkaloids in microbes is limited. Recently, de novo production of the MIA alkaloid strictosidine has been achieved in yeast by introducing 21 new genes and deleting 3 genes in the yeast genome (Table 1) [25]. Glucosinolates are sulfur-rich, amino acid-derived natural compounds. Tryptophan-derived indolylglucosinolate (IG) has recently been produced in S. cerevisiae by inserting eight plant genes into the genome, which represents a rare example of glucosinolate production in microbes (Figure 1) [26]. Although plant platforms are more suitable for scalable alkaloid production, metabolic engineering in microbes has shown potential capability for cost-efficient production of these plant-derived compounds [7,25].

\section{Terpenoids}

Terpenoids are present in green foods, soy plants and cereals, and serve as the largest class of phytonutrients with anti-inflammatory, anti-infectious and anti-cancer properties [3,7]. Terpenoids are isopentenyl pyrophosphate (IPP) or dimethylallyl pyrophosphate (DMAPP) derived compounds of diverse carbon skeletons, including monoterpenes $\left(\mathrm{C}_{10}\right.$, e.g. menthol), sesquiterpenes $\left(C_{15}\right.$, e.g. artemisinin $)$, diterpenes $\left(C_{20}\right.$, e.g. paclitaxel $)$, triterpens $\left(C_{30}\right.$, e.g. steroids $)$, tetraterpenes $\left(\mathrm{C}_{40}\right.$, carotenoids) and polyterpenes [5]. Microbial production of terpenoids such as taxadiene (intermediate of anticancer drug paclitaxel) and artemisinic acid (precursor of antimalarial drug artemisinin) exemplified the advances and successes of metabolic engineering for production of value-added terpenoid pharmaceuticals. In nutraceutical industries, tetraterpene carotenoids such as $\beta$-carotene, lutein, lycopene, $\alpha$-carotene and astaxanthin serve as natural food colorants and feed supplements [5]. Combinatorial carotenoid biosynthesis in heterologous non-carotenogenic hosts like E. coli and S. cerevisiae has been achieved for decades. Since then, metabolic engineering efforts have mainly focused on large-scale production of carotenoids. Strain optimization via systematic and combinatorial gene knockouts significantly increased lycopene production in E. coli [6]. Engineering central metabolic modules of E. coli to increase ATP and NADPH supplies improved $\beta$-carotene production to $2.1 \mathrm{~g} / \mathrm{L} \beta$-carotene with a yield of $60 \mathrm{mg} / \mathrm{g}$ DCW [27]. Lycopene derived zeaxanthin was biosynthesized and its production improved to $43.46 \mathrm{mg} / \mathrm{L}$ via protein fusion approaches for coordinating expression of lycopene $\beta$-cyclase gene $\operatorname{crt} Y$ and $\beta$-carotene 3-hydroxylase gene crtZ [28]. A plasmid-free E. coli strain with the xanthophyll biosynthetic genes chromosomally integrated produced astaxanthin with a yield of 1.4 $\mathrm{mg} / \mathrm{g}$ DCW [29]. Besides conventional carotenoids, discovery and production of new carotenoids with health-promoting benefits have also increased the pool of available carotenoids. Novel carotenoids such as 4-ketozeinoxanthin and the rare $\mathrm{C}_{50}$ carotenoids sarcinaxanthin, decaprenoxanthin and sarprenoxanthin have been produced in lycopene-producing $E$. coli hosts [30,31]. Recently, a non-natural purple carotenoid $\mathrm{C}_{50}$-astaxanthin was produced in E. coli using mutants of the first two pathway enzymes, farnesyl diphosphate synthase (FDS) and carotenoid synthase (CrtM), with shifted size-range substrate selectivities to enhance production of $\mathrm{C}_{50}$ backbone while suppress non-target compounds [32].

\section{Prebiotics}

A prebiotic is defined as "a nonviable food component that confers a health benefit on the host associated with modulation of the microbiota" by The Food and Agriculture Organization of the 
United Nations (FAO) [33]. Prebiotics are non-digestible saccharide polymers that contain 3-10 monomeric sugar units (Figure 2). The beneficial effects of prebiotics on humans lie in their modulation on diversity and metabolic activity of the gut microbiota, which may consequently have significant impact on the host immune system. Thus, prebiotics could be used to treat inflammatory diseases by boosting probiotics like Bifidobacteria or Lactobacillus species within the gut microbiota [34]. Representative prebiotics include inulin, fructo-oligosaccharides (FOS) and galacto-oligosaccharides (GOS). Inulin and inulin-type fructans, also known as soluble dietary fibres, could be produced by probiotic Lactobacillus gasseri strains [35]. GOS are lactose-derived galactose polymers with shorter polymer chains. GOS could be produced by Kluyveromyces lactis with a titer of $177 \mathrm{~g} / \mathrm{L}$ from $400 \mathrm{~g} / \mathrm{L}$ lactose [36]. Expression of codon-optimized $\beta$-galactosidase from the hyperthermophile Sulfolobus solfataricus in Lactococcus lactis efficiently converted lactose to GOS [37]. 2'-fucosyllactose (2'-FL) is one of the most abundant oligosaccharides in human milk and has been approved as nutritional additive in term infant and toddler formulas. By increasing the availability GDP-L-fucose and overexpression of fucosyltransferase, 2'-FL could be largely produced from lactose and glycerol in E. coli $[38,39]$.

\section{Polysaccharides}

Polysaccharides are sugar polymers of versatile structures that can be largely produced by several bacteria, yeast and fungi or extracted from plant and animal tissues (Figure 2). Microbial polysaccharides have been recognized as a source for nutraceuticals owing to their health-beneficial properties. Bacterial polysaccharides such as xanthan, gellan, dextrans and alginate can be commercialized via microbial production and purification [40]. Exopolysaccharides (EPS) are preferentially produced via metabolic engineering of Lactococcus and Streptococcus for their wide use in dairy products. Especially, bioactive fungal polysaccharides have demonstrated extensive immunostimulating, antitumor, antimicrobial, antioxidant, hypocholesterolaemic and hypoglycaemic benefits, thus indicating great potential in both nutraceutical and pharmaceutical applications [41]. Potential antitumor and antiviral polysaccharide scleroglucan is an extracellular glucan excreted by mycelia of Sclerotium rolfsii, whose yields could be enhanced via addition of L-lysine and uridine mono-phosphate (UMP) [41]. Instead of extraction from animal tissues, several animal polysaccharides like hyaluronic acid (HA), chondroitin and heparosan have also been produced by microbial hosts. Microbial production of hyaluronic acid has been achieved in various hosts including E. coli, Lactococcus lactis and Streptomyces albulus [42-44]. Medicinally important polysaccharides such as heparosan and chondroitin can be produced by engineered $E$. coli reaching a relatively high titer of $1.88 \mathrm{~g} / \mathrm{L}$ and $2.4 \mathrm{~g} / \mathrm{L}$, respectively (Table 1) [45,46].

\section{Poly amino acids}

Poly amino acids are synthesized in microorganisms from one or two type of amino acids by ribosome-independent enzymatic processes that distinguish them from protein synthesis. Three kinds of poly amino acids, namely poly- $\gamma$-glutamic acid $(\gamma$-PGA), poly- $\varepsilon$-L-lysine $(\varepsilon-\mathrm{PL})$ and multi-L-arginyl-poly(L-aspartic acid) (cyanophycin), are present in nature (Figure 2). $\gamma$-PGA is a water-soluble and biodegradable polymer that can be used as a degradable biomaterial such as drug carriers or hydrogels. $\gamma$-PGA can be largely produced $(20-50 \mathrm{~g} / \mathrm{L})$ by Bacillus species when 
feeding L-glutamic acid. In order to achieve $\gamma$-PGA production from glucose, optimization of co-cultivation of Corynebacterium glutamicum and Bacillus subtilis could maximally produce $32.8 \mathrm{~g} / \mathrm{L} \gamma$-PGA [47]. Cyanophycin was produced from equimolar amounts of arginine and aspartic acid by cyanobacteria and some other chemotrophic bacteria (e. g. Acinetobacter calcoaceticus), which can be used as a precursor of dipeptides for nutritional and therapeutic applications. Overexpression of cyanophycin synthetase cphA from Synechocystis sp. PCC 6803 in E. coli achieved cyanophycin production with productivity of $120 \mathrm{mg} / \mathrm{g}$ CDW [48]. $\varepsilon-\mathrm{PL}$ is a microbial-derived homo-poly amino acid that is synthesized by polymerization of lysine via $\varepsilon-\mathrm{PL}$ synthetase (PLS). $\varepsilon$-PL possesses antibacterial and anticancer activities and has been approved as food preservative or dietary agent in Japan and United States [49]. $\varepsilon$-PL was produced by Streptomyces species such as Streptomyces albulus. Production of $\varepsilon$-PL in Streptomyces sp. $\mathrm{M}-\mathrm{Z} 18$ could reach $35.14 \mathrm{~g} / \mathrm{L}$ when using glucose and glycerol as carbon sources [64].

\section{Conclusions}

The last few decades have witnessed the remarkable progresses of nutraceuticals production via metabolic engineering of microbial-based platforms, which is in response to the great demands for value-added nutraceuticals to fortify humans against diseases. Metabolic engineering in microbes made possible not only the laboratory-scale preparation but also industrial-scale production of complex natural products such as carotenoids from simple carbon sources. Especially, with the elucidation of biosynthetic pathways and development of synthetic biology strategies, novel or more complicated value-added compounds could be produced in microbes in a naturally developed manner. The amenability of genetic manipulation and tolerance of heterologous enzymes enabled microbes to produce diverse nutraceuticals via harnessing their native metabolic networks. One of the major limitations of microbial platform is the efficiency of plant or animal-derived enzymes that limit the productivity of the target compounds. While other non-microbial production platforms such as algae and plant-derived cell cultures have also been developed to produce natural or non-natural value-added nutraceuticals, there is little doubt that continuing advances in metabolic engineering and synthetic biology will allow the production of virtually any nutraceutical through a microbial host.

\section{ACKNOWLEDGMENTS}

Mattheos Koffas acknowledges partial financial support from NSF grant number MCB-1448657.

Table 1. Biological production of nutraceuticals via metabolic engineering in different hosts

\begin{tabular}{lllll}
\hline Nutraceuticals & Heterologous hosts & Fed precursors & Titers (mg/L) & References \\
\hline Polyphenolic compounds & & & & \\
\hline Apigenin (Flavone) & E. coli & Tyrosine & 13 & {$[9]$} \\
Chrysin (Flavone) & E. coli & Phenylalanine & 9.4 & {$[9]$} \\
Pinocembrin (Flavanone) & E. coli & Glucose & 40.2 & {$[15]$} \\
Eriodictyol (Flavanone) & E. coli & Tyrosine & 107 & {$[51]$} \\
Naringenin (Flavanone) & S. cerevisiae & Glucose & 108.9 & {$[52]$} \\
Catechin (Flavanonol) & E. coli & Eriodictyol & 910.9 & {$[53]$} \\
Genistein (Isoflavonone) & E. coli-S. cerevisiae & Tyrosine & 6 & {$[54]$} \\
Resveratrol (Stilbenoid) & E. coli & Tyrosine & 35.02 & {$[55]$} \\
\hline
\end{tabular}




\begin{tabular}{|c|c|c|c|c|}
\hline Pinosylvin (Stilbenoid) & E. coli & Glucose & 70 & {$[16]$} \\
\hline Curcumin (Curcuminoid) & E. coli & Tyrosine & 0.6 & {$[56]$} \\
\hline Caffeic acid & E. coli & $\begin{array}{l}\text { Glucose } \\
\text { glycerol }\end{array}$ & 766.68 & {$[57]$} \\
\hline \multicolumn{5}{|l|}{ Flavonoid derivatives } \\
\hline Sakuranetin & E. coli & Glucose & 42.5 & {$[17]$} \\
\hline Ponciretin & E. coli & Glucose & 40.1 & {$[17]$} \\
\hline 7-O-Methyl aromadendrin & E. coli & p-coumaric acid & 2.7 & {$[58]$} \\
\hline Luteolin 4-O-glucosides & E. coli & Luteolin & 10.86 & {$[10]$} \\
\hline Luteolin 7-O-glucosides & E. coli & Luteolin & 6.52 & {$[10]$} \\
\hline Luteolin-7-O-glucuronide & E. coli & Luteolin & 300 & {$[11]$} \\
\hline Kaempferol 3-O-glucoside & E. coli & Naringenin & 109.3 & {$[10]$} \\
\hline Kaempferol-3-O-rhamnoside & E. coli & Kaempferol & 150 & {$[12]$} \\
\hline Quercetin 3-O-glucoside & E. coli & Quercetin & 11.54 & {$[10]$} \\
\hline Quercetin-3-O-glucuronide & E. coli & Quercetin & 687 & {$[11]$} \\
\hline Quercetin-3-O-rhamnoside & E. coli & Quercetin & 200 & {$[12]$} \\
\hline Anthocyanin & E. coli & Catechin & 110 & {$[59]$} \\
\hline Genistein glucosides & E. coli & Genistein & 37.29 & {$[60]$} \\
\hline Genistin & S. cerevisiae & Genistein & - & {$[61]$} \\
\hline Daidzin & S. cerevisiae & Daidzein & - & {$[8]$} \\
\hline 3-Hydroxydaidzein & E. coli & Daidzein & 75 & {$[61]$} \\
\hline \multicolumn{5}{|l|}{ Alkaloids } \\
\hline \multicolumn{5}{|l|}{ (Benzylisoquinoline alkaloid) } \\
\hline $\begin{array}{l}\text { Strictosidine (Monoterpene } \\
\text { indole alkaloid) }\end{array}$ & S. cerevisiae & Glucose & 0.53 & {$[25]$} \\
\hline $\begin{array}{l}\text { Indolylglucosinolate } \\
\text { (Glucosinolate) }\end{array}$ & S. cerevisiae & Glucose & 1.07 & {$[26]$} \\
\hline \multicolumn{5}{|l|}{ Terpenoids } \\
\hline Lycopene & E. coli & Glucose & $18 \mathrm{mg} / \mathrm{g}$ & {$[62]$} \\
\hline$\beta$-carotene & E. coli & Glucose & 2100 & {$[27]$} \\
\hline Zeaxanthin & E. coli & Glucose & 43.46 & {$[28]$} \\
\hline Astaxanthin & E. coli & Glucose & $1.4 \mathrm{mg} / \mathrm{g}$ & {$[29]$} \\
\hline \multicolumn{5}{|l|}{ Prebiotics } \\
\hline 2-Fucosyllactose & E. coli & $\begin{array}{l}\text { Lactose } \\
\text { glycerol }\end{array}$ & 20280 & {$[39]$} \\
\hline Galactooligosaccharides & Lactococcus lactis & Lactose & 197000 & {$[37]$} \\
\hline \multicolumn{5}{|l|}{ Polysaccaharides } \\
\hline Heparosan & E. coli & Glycerol & 1880 & {$[46]$} \\
\hline Hyaluronan & Streptomyces albulus & Sucrose & 6200 & {$[42]$} \\
\hline \multirow[t]{2}{*}{ Scleroglucan } & Sclerotium rolfsii & Uridine & 22320 & {$[63]$} \\
\hline & & Mono-phosphate & & \\
\hline Chondroitin & E. coli & Glucose & 2400 & {$[45]$} \\
\hline
\end{tabular}

Poly amino acids 


\begin{tabular}{llllll}
\hline Poly- $\varepsilon$-L-lysine & Streptomyces & Glucose & and & 35140 & [64] \\
sp.M-Z18 & glycerol & & & \\
Bacillus subtilis BL53 & $\begin{array}{l}\text { L-glutamic } \\
\text { and glycerol }\end{array}$ & acid & 17000 & {$[65]$} \\
Cyanophycin & E. coli & Yeast extract & $120 \mathrm{mg} / \mathrm{g}$ & [48] \\
\hline
\end{tabular}

Figure 1. Examples of microbial-based production of phytochemicals from aromatic amino acids. Arrows represent the metabolic flux from primary shikimate pathway to target phytochemicals. Polyphenolic compounds are derived from phenylalanine or tyrosine, benzylisoquinoline alkaloids (BIA) are derived from tyrosine, monoterpene indole alkaloids (MIA) and indolylglucosinolate (IG) are derived from tryptophan.

Figure 2. Representatives of microbial-produced prebiotics, polysaccaharides and poly amino acids.

\section{References and recommended reading}

Papers of particular interest, published within the period of review, have been highlighted as:

.. of outstanding interest

1. DeFelice SL: The nutraceutical revolution: its impact on food industry R\&D. Trends Food Sci Technol 1995, 6:59-61.

2. Pandey M, Verma RK, Saraf SA: Nutraceuticals: new era of medicine and health. Asian J Pharm Clin Res 2010, 3:11-15.

3. Jain N, Ramawat KG: Nutraceuticals and antioxidants in prevention of diseases. Natural

4. De Luca V, Salim V, Atsumi SM, Yu F: Mining the biodiversity of plants: a revolution in the making. Science 2012, 336:1658-1661.

5. Marienhagen J, Bott M: Metabolic engineering of microorganisms for the synthesis of plant natural products. J Biotechnol 2013, 163:166-178.

6. Lin Y, Jain R, Yan Y: Microbial production of antioxidant food ingredients via metabolic engineering. Curr Opin Biotechnol 2014, 26:71-78.

7. Mora-Pale M, Sanchez-Rodriguez SP, Linhardt RJ, Dordick JS, Koffas MA: Metabolic engineering and in vitro biosynthesis of phytochemicals and non-natural analogues. Plant Sci 2013, 210:10-24.

8. Leonard E, Koffas MA: Engineering of artificial plant cytochrome P450 enzymes for synthesis of isoflavones by Escherichia coli. Appl Environ Microbiol 2007, 73:7246-7251.

9. Miyahisa I, Funa N, Ohnishi Y, Martens S, Moriguchi T, Horinouchi S: Combinatorial biosynthesis of flavones and flavonols in Escherichia coli. Appl Microbiol Biotechnol 2006, 
71:53-58.

10. He X-Z, Li W-S, Blount JW, Dixon RA: Regioselective synthesis of plant (iso) flavone glycosides in Escherichia coli. Appl Microbiol Biotechnol 2008, 80:253-260.

11. Kim SY, Lee HR, Park K-s, Kim B-G, Ahn J-H: Metabolic engineering of Escherichia coli Microbiol Biotechnol 2015, 99:2233-2242.

12. Kim B-G, Kim HJ, Ahn J-H: Production of bioactive flavonol rhamnosides by expression of plant genes in Escherichia coli. J Agric Food Chem 2012, 60:11143-11148.

13. $\mathrm{Xu}$ P, Ranganathan $\mathrm{S}$, Fowler $\mathrm{ZL}$, Maranas CD, Koffas MA: Genome-scale metabolic network modeling results in minimal interventions that cooperatively force carbon flux towards malonyl-CoA. Metab Eng 2011, 13:578-587.

14. Yang Y, Lin Y, Li L, Linhardt RJ, Yan Y: Regulating malonyl-CoA metabolism via synthetic antisense RNAs for enhanced biosynthesis of natural products. Metab Eng 2015, 29:217-226.

The authors demonstrated the effectiveness of asRNA as a powerful tool to achieve malonyl-CoA enrichment via down-regulating fatty acid biosynthesis in Escherichia coli, which led to improved production of natural products 4-hydroxycoumarin, resveratrol and naringenin.

15. $\mathrm{Wu} \mathrm{J}, \mathrm{Du} \mathrm{G}$, Zhou J, Chen $\mathrm{J}$ : Metabolic engineering of Escherichia coli for (2S)-pinocembrin production from glucose by a modular metabolic strategy. Metab Eng 2013, 16:48-55.

16. van Summeren-Wesenhagen PV, Marienhagen J: Metabolic engineering of Escherichia coli for the synthesis of the plant polyphenol pinosylvin. Appl Environ Microbiol 2015, 81:840-849.

17. Kim M-J, Kim B-G, Ahn J-H: Biosynthesis of bioactive O-methylated flavonoids in Escherichia coli. Appl Microbiol Biotechnol 2013, 97:7195-7204.

18. Bhan BFC, R. J. Linhardt, M.A.G. Koffas: Expanding the chemical space of polyketides through structure-guided mutagenesis of Vitis vinifera stilbene synthase. Biochimie 2015.

Demonstration of the application of enzyme promiscuity and the feasibility of rational enzyme engineering of stilbene synthase to produce multiple non-natural polyketides.

19. Bhan N, Li L, Cai C, Xu P, Linhardt RJ, Koffas MA: Enzymatic formation of a resorcylic acid by creating a structure-guided single-point mutation in stilbene synthase. Protein Sci 2015, 24:167-173.

20. Nakagawa A, Minami H, Kim J-S, Koyanagi T, Katayama T, Sato F, Kumagai H: A bacterial platform for fermentative production of plant alkaloids. Nat Commun 2011, 2:326.

The authors established a bacterial platform for plant alkaloid (S)-reticuline production (46 mg/L) from simple carbon source glycerol, suggesting its feasibility as a fermentation platform for low-cost production of diverse plant alkaloids.

21. Fossati E, Ekins A, Narcross L, Zhu Y, Falgueyret J-P, Beaudoin GA, Facchini PJ, Martin VJ: Reconstitution of a 10-gene pathway for synthesis of the plant alkaloid dihydrosanguinarine in Saccharomyces cerevisiae. Nat Commun 2014, 5.

22. Nakagawa A, Matsuzaki C, Matsumura E, Koyanagi T, Katayama T, Yamamoto K, Sato F, Kumagai H, Minami H: (R, S)-Tetrahydropapaveroline production by stepwise fermentation using engineered Escherichia coli. Sci Rep 2014, 4.

23. Minami H, Kim J-S, Ikezawa N, Takemura T, Katayama T, Kumagai H, Sato F: Microbial 
production of plant benzylisoquinoline alkaloids. Proc Natl Acad Sci U S A 2008, 105:7393-7398.

24. Hawkins KM, Smolke CD: Production of benzylisoquinoline alkaloids in Saccharomyces cerevisiae. Nat Chem Biol 2008, 4:564-573.

25. Brown S, Clastre M, Courdavault V, O'Connor SE: De novo production of the plant-derived alkaloid strictosidine in yeast. Proc Natl Acad Sci U S A 2015, 112:3205-3210.

35. Anwar MA, Kralj S, Piqué AV, Leemhuis H, van der Maarel MJ, Dijkhuizen L: Inulin and

First report of de novo production of a plant-derived monoterpene indole alkaloid strictosidine $(0.5$ $\mathrm{mg} / \mathrm{L}$ ) in yeast by introducing a 21-gene pathway and deleting 3 genes of yeast.

26. Mikkelsen MD, Buron LD, Salomonsen B, Olsen CE, Hansen BG, Mortensen UH, Halkier BA: Microbial production of indolylglucosinolate through engineering of a multi-gene pathway in a versatile yeast expression platform. Metab Eng 2012, 14:104-111.

First production of plant-derived glucosinolates in a stable and robust microbial host by intergrating multi-gene pathways (up to 22 genes) into the yeast genome.

27. Zhao J, Li Q, Sun T, Zhu X, Xu H, Tang J, Zhang X, Ma Y: Engineering central metabolic modules of Escherichia coli for improving 及-carotene production. Metab Eng 2013, 17:42-50.

28. Li X-R, Tian G-Q, Shen H-J, Liu J-Z: Metabolic engineering of Escherichia coli to produce

29. Lemuth K, Steuer K, Albermann C: Engineering of a plasmid-free Escherichia coli strain for improved in vivo biosynthesis of astaxanthin. Microb Cell Fact 2011, 10:29.

30. Netzer R, Stafsnes MH, Andreassen T, Goksøyr A, Bruheim P, Brautaset T: Biosynthetic pathway for $\gamma$-cyclic sarcinaxanthin in Micrococcus luteus: Heterologous expression and evidence for diverse and multiple catalytic functions of C50 carotenoid cyclases. $J$ Bacteriol 2010, 192:5688-5699.

31. Maoka T, Takemura M, Tokuda H, Suzuki N, Misawa N: 4-Ketozeinoxanthin, a novel carotenoid produced in Escherichia coli through metabolic engineering using carotenogenic genes of bacterium and liverwort. Tetrahedron Lett 2014, 55:6708-6710.

32. Furubayashi M, Ikezumi M, Takaichi S, Maoka T, Hemmi H, Ogawa T, Saito K, Tobias AV, Umeno D: A highly selective biosynthetic pathway to non-natural C50 carotenoids assembled from moderately selective enzymes. Nat Commun 2015, 6.

The authors established a pathway that selectively produced non-natural carotenoid $\mathrm{C}_{50}$-astaxanthin by screening variants of the first two enzymes with shifted substrate specificity and combining them to channel precursors to $\mathrm{C}_{50}$ backbones.

33. Pineiro M, Asp N-G, Reid G, Macfarlane S, Morelli L, Brunser O, Tuohy K: FAO Technical meeting on prebiotics. J Clin Gastroenterol 2008, 42:S156-S159. Lin C-S, Chang C-J, Lu C-C, Martel J, Ojcius DM, Ko Y-F, Young JD, Lai H-C: Impact of the gut microbiota, prebiotics, and probiotics on human health and disease. Biomed $J$ 2014, 37:259. levan synthesis by probiotic Lactobacillus gasseri strains: characterization of three novel fructansucrase enzymes and their fructan products. Microbiology 2010, 156:1264-1274. Jimenez-Barbero J, Haltrich D, Ballesteros Olmo AO, Fernandez-Lobato M, Plou FJ: 
Production of galacto-oligosaccharides by the $\beta$-galactosidase from Kluyveromyces lactis: Comparative analysis of permeabilized cells versus soluble enzyme. J Agric Food Chem 2011, 59:10477-10484.

37. Yu L, O'Sullivan D: Production of galactooligosaccharides using a hyperthermophilic B-galactosidase in permeabilized whole cells of Lactococcus lactis. J Dairy Sci 2014, 97:694-703.

38. Lee W-H, Pathanibul P, Quarterman J, Jo J-H, Han NS, Miller MJ, Jin Y-S, Seo J-H: Whole cell biosynthesis of a functional oligosaccharide, 2'-fucosyllactose, using engineered Escherichia coli. Microb Cell Fact 2012, 11:48.

39. Baumgärtner F, Seitz L, Sprenger GA, Albermann C: Construction of Escherichia coli strains with chromosomally integrated expression cassettes for the synthesis of 2'-fucosyllactose. Microb Cell Fact 2013, 12:40.

First establishment of selection marker-free $E$. coli strain that produces 2 '-FL with a final titer of 20.28 $\mathrm{g} / \mathrm{L}$ in fed-batch fermentation from lactose and glycerol.

40. Giavasis I: Production of microbial polysaccharides for use in food. Microbial Production of Food Ingredients, Enzymes and Nutraceuticals. Woodhead Publishing; 2013:413-468.

41. Giavasis I: Bioactive fungal polysaccharides as potential functional ingredients in food and nutraceuticals. Curr Opin Biotechnol 2014, 26:162-173.

42. Yoshimura T, Shibata N, Hamano Y, Yamanaka K: Heterologous production of hyaluronic acid in an e-poly-L-lysine producer, Streptomyces albulus. Appl Environ Microbiol 2015, 81:3631-3640.

43. Yu H, Stephanopoulos G: Metabolic engineering of Escherichia coli for biosynthesis of hyaluronic acid. Metab Eng 2008, 10:24-32.

44. Sheng J, Ling $\mathrm{P}$, Wang F: Constructing a recombinant hyaluronic acid biosynthesis operon and producing food-grade hyaluronic acid in Lactococcus lactis. J Ind Microbiol Biotechnol 2015, 42:197-206.

45. He W, Fu L, Li G, Jones JA, Linhardt RJ, Koffas M: Production of chondroitin in metabolically engineered $\boldsymbol{E}$. coli. Metab Eng 2015, 27:92-100.

First successful demonstration of high-titer production of chondroitin $(2.4 \mathrm{~g} / \mathrm{L})$ in a non-pathogenic $E$. coli strain that opens the gates for production of chondroitin-derived polysaccharides with nutraceutical or pharmaceutical applications.

46. Zhang C, Liu L, Teng L, Chen J, Liu J, Li J, Du G, Chen J: Metabolic engineering of Escherichia coli BL21 for biosynthesis of heparosan, a bioengineered heparin precursor. Metab Eng 2012, 14:521-527.

47. Shi F, Xu Z, Cen P: Microbial production of natural poly amino acid. Sci China Ser B 2007, 50:291-303.

48. Tseng WC, Fang TY, Cho CY, Chen PS, Tsai CS: Assessments of growth conditions on the production of cyanophycin by recombinant Escherichia coli strains expressing cyanophycin synthetase gene. Biotechnol Progr 2012, 28:358-363.

49. El-Sersy NA, Abdelwahab AE, Abouelkhiir SS, Abou-Zeid DM, Sabry SA: Antibacterial and anticancer activity of $\varepsilon$-poly-L-lysine ( $\varepsilon$-PL) produced by a marine Bacillus subtilis sp. J Basic Microbiol 2012, 52:513-522.

50. Kroll J, Klinter S, Steinbüchel A: A novel plasmid addiction system for large-scale production of cyanophycin in Escherichia coli using mineral salts medium. Appl 
Microbiol Biotechnol 2011, 89:593-604.

51. Zhu S, Wu J, Du G, Zhou J, Chen J: Efficient synthesis of eriodictyol from L-tyrosine in Escherichia coli. Appl Environ Microbiol 2014, 80:3072-3080.

52. Koopman F, Beekwilder J, Crimi B, van Houwelingen A, Hall RD, Bosch D, van Maris A, Pronk JT, Daran J-M: De novo production of the flavonoid naringenin in engineered Saccharomyces cerevisiae. Microb Cell Fact 2012, 11:155.

First report of de novo production of naringenin in yeast from glucose via optimization of the product pathway, improvement of precursor supply and reduction of byproducts.

53. Zhao S, Jones JA, Lachance DM, Bhan N, Khalidi O, Venkataraman S, Wang Z, Koffas MA: Improvement of catechin production in Escherichia coli through combinatorial metabolic engineering. Metab Eng 2015, 28:43-53.

54. Katsuyama Y, Miyahisa I, Funa N, Horinouchi S: One-pot synthesis of genistein from tyrosine by coincubation of genetically engineered Escherichia coli and Saccharomyces cerevisiae cells. Appl Microbiol Biotechnol 2007, 73:1143-1149.

55. $\mathrm{Wu}$ J, Liu P, Fan Y, Bao H, Du G, Zhou J, Chen J: Multivariate modular metabolic engineering of Escherichia coli to produce resveratrol from L-tyrosine. J Biotechnol 2013, 167:404-411.

56. Wang S, Zhang S, Xiao A, Rasmussen M, Skidmore C, Zhan J: Metabolic engineering of Escherichia coli for the biosynthesis of various phenylpropanoid derivatives. Metab Eng 2015, 29:153-159.

57. Huang Q, Lin Y, Yan Y: Caffeic acid production enhancement by engineering a phenylalanine over-producing Escherichia coli strain. Biotechnol Bioeng 2013, 110:3188-3196.

58. Malla S, Koffas MA, Kazlauskas RJ, Kim B-G: Production of 7-O-methyl aromadendrin, a medicinally valuable flavonoid, in Escherichia coli. Appl Environ Microbiol 2012, 78:684-694.

59. Leonard E, Yan Y, Fowler ZL, Li Z, Lim C-G, Lim K-H, Koffas MA: Strain improvement of recombinant Escherichia coli for efficient production of plant flavonoids. Mol Pharm 2008, 5:257-265.

60. Pandey RP, Parajuli P, Koirala N, Lee JH, Park YI, Sohng JK: Glucosylation of isoflavonoids in engineered Escherichia coli. Mol Cells 2014, 37:172.

61. Li J, Li Z, Li C, Gou J, Zhang Y: Molecular cloning and characterization of an isoflavone 7-O-glucosyltransferase from Pueraria lobata. Plant Cell Rep 2014, 33:1173-1185.

62. Alper H, Miyaoku K, Stephanopoulos G: Construction of lycopene-overproducing $\boldsymbol{E}$. coli strains by combining systematic and combinatorial gene knockout targets. Nat Biotechnol 2005, 23:612-616.

63. Survase SA, Saudagar PS, Singhal RS: Enhanced production of scleroglucan by Sclerotium rolfsii MTCC 2156 by use of metabolic precursors. Bioresour Technol 2007, 98:410-415.

64. Chen X-S, Ren X-D, Dong N, Li S, Li F, Zhao F-L, Tang L, Zhang J-H, Mao Z-G: Culture medium containing glucose and glycerol as a mixed carbon source improves E-poly-L-lysine production by Streptomyces sp. M-Z18. Bioprocess Biosyst Eng 2012, 35:469-475.

65. Da Silva SB, Cantarelli VV, Ayub MAZ: Production and optimization of poly- $\gamma$-glutamic acid by Bacillus subtilis BL53 isolated from the Amazonian environment. Bioprocess 
465

Biosyst Eng 2014, 37:469-479.

466

467

468 
Figure 1

470

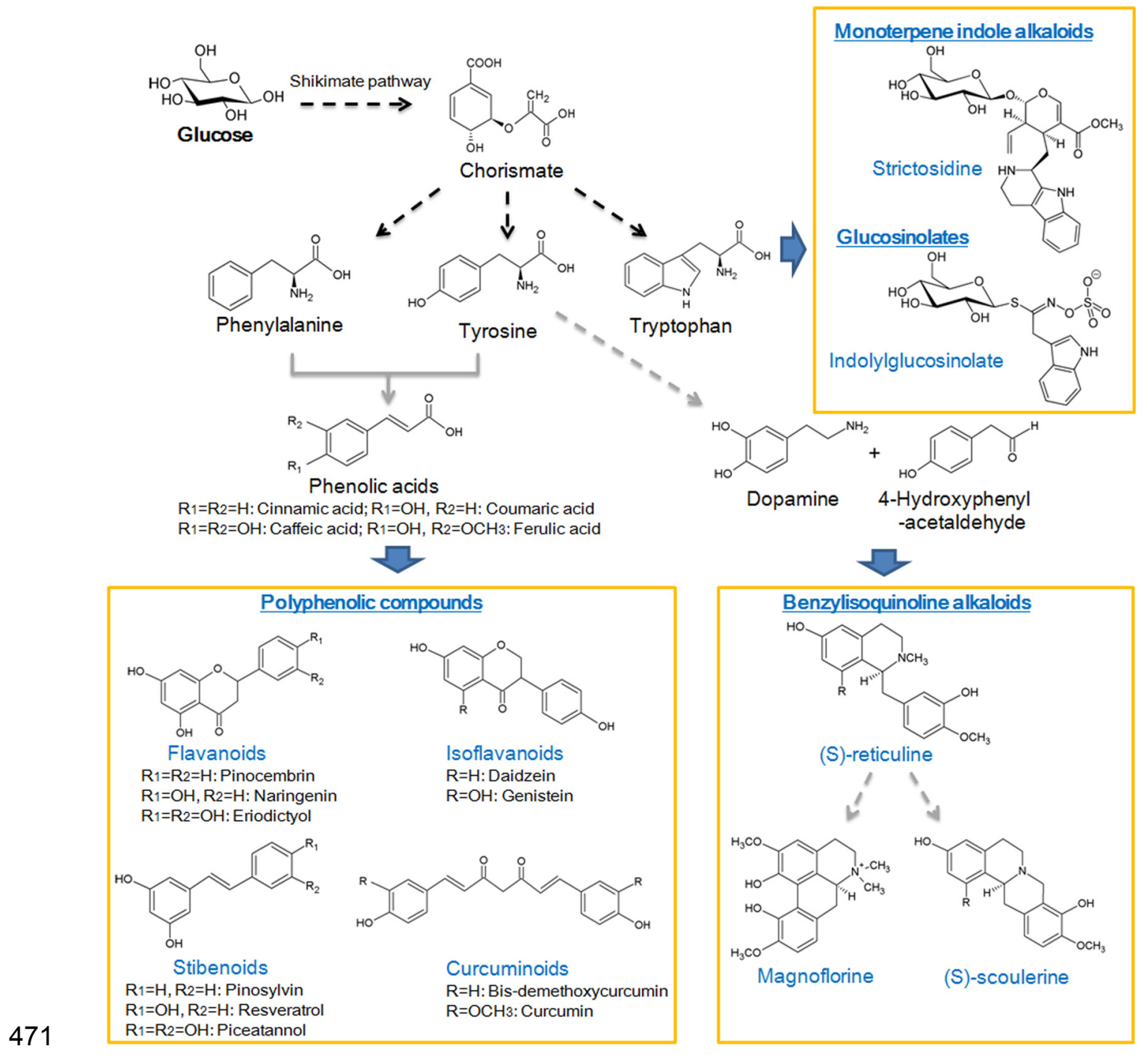


Figure 2

474

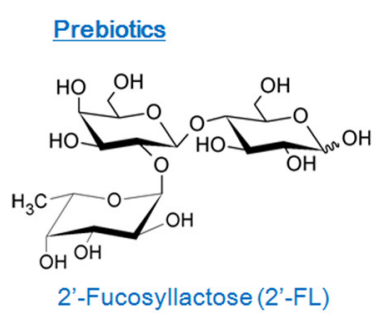

Polysaccharides

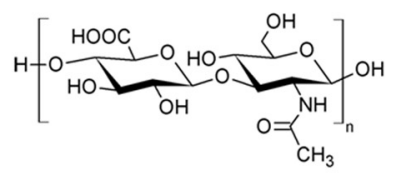

Hyaluronic acid

Poly amino acids

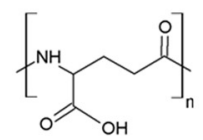

475

Poly-Y-glutamic acid (

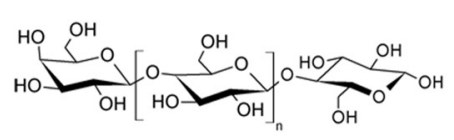

Galacto-oligosaccharides (GOS)

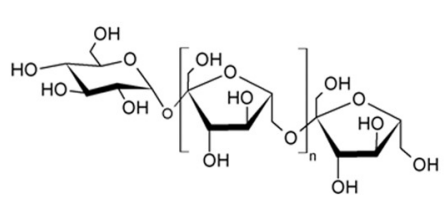

Fructo-oligosaccharides (FOS)

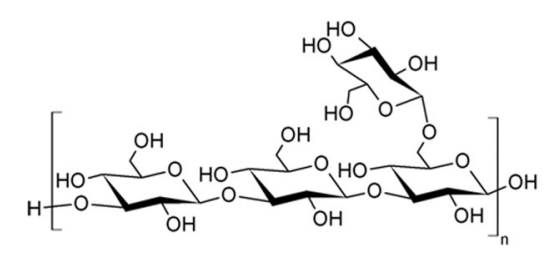

Scleroglucan polysaccharide

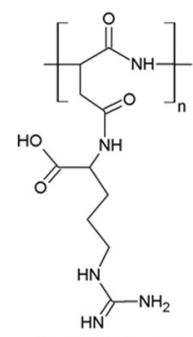

Cyanophycin

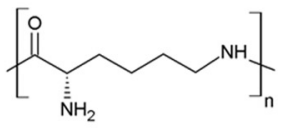

Poly- $\varepsilon$-L-lysine ( $\varepsilon-P L)$ 
477 Graphical Abstract

478

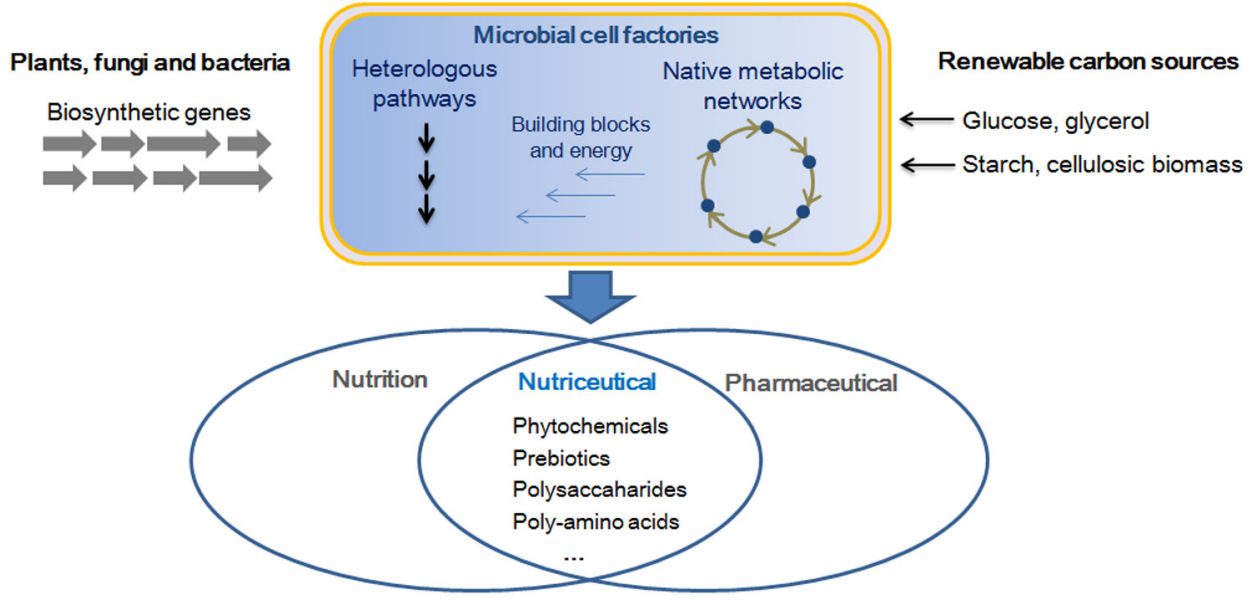

